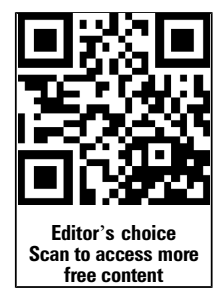

\title{
ASPECTS decay during inter-facility transfer predicts patient outcomes in endovascular reperfusion for ischemic stroke: a unique assessment of dynamic physiologic change over time
}

\author{
Chung-Huan J Sun, ${ }^{1}$ Kerrin Connelly, ${ }^{2}$ Raul G Nogueira, ${ }^{1,2}$ Brenda A Glenn, ${ }^{3}$ \\ Susan Zimmermann, ${ }^{3}$ Kim Anda, ${ }^{4}$ Deborah Camp, ${ }^{5}$ Susan Gaunt, ${ }^{6}$ Herma Pallard, ${ }^{6}$ \\ Michele Eckenroth, ${ }^{7}$ Michael R Frankel, ${ }^{1,2}$ Samir R Belagaje, ${ }^{1,2}$ Aaron M Anderson, ${ }^{1,2}$ \\ Fadi Nahab, ${ }^{1}$ Manuel Yepes, ${ }^{1}$ Rishi Gupta ${ }^{3}$
}

For numbered affiliations see end of article.

\section{Correspondence to Dr R Gupta, Wellstar Neurosurgery, 61 Whitcher Street, Suite 3110, Marietta, GA 30060, USA; guptar31@gmail.com}

Received 23 November 2013 Revised 27 December 2013 Accepted 30 December 2013 Published Online First 17 January 2014

\section{ABSTRACT}

Background Pretreatment Alberta Stroke Program Early CT Scores (ASPECTS) is associated with clinical outcomes. The rate of decline between subsequent images, however, may be more predictive of outcomes as it integrates time and physiology.

Methods A cohort of patients transferred from six primary stroke centers and treated with intra-arterial therapy (IAT) was retrospectively studied. Absolute ASPECTS decay was defined as ((ASPECTS ${ }_{\text {First } \mathrm{CT}}$ ASPECTS second $\mathrm{CT}$ )/hours elapsed between images). A logistic regression model was performed to determine if the rate of ASPECTS decay predicted good outcomes at 90 days (modified Rankin Scale score of $0-2$ ).

Results 106 patients with a mean age of $66 \pm 14$ years and a median National Institutes of Health Stroke Scale score of 19 (IQR 15-23) were analyzed. Median time between initial CT at the outside hospital to repeat $C T$ at our facility was $2.7 \mathrm{~h}$ (IQR 2.0-3.6). Patients with good outcomes had lower rates of absolute ASPECTS decay compared with those who did not $(0.14 \pm 0.23$ score/h vs $0.49 \pm 0.39$ score/h; $p<0.001)$. In multivariable modeling, the absolute rate of ASPECTS decay (OR 0.043; $95 \% \mathrm{Cl}$ 0.004 to $0.471 ; p=0.01$ ) was a stronger predictor of good patient outcome than static pretreatment ASPECTS obtained before IAT (OR 0.64; 95\% Cl 0.38 to 1.04; $\mathrm{p}=0.075)$. In practical terms, every 1 unit increase in ASPECTS decline per hour correlates with a 23-fold lower probability of a good outcome.

Conclusions Patients with faster rates of ASPECTS decay during inter-facility transfers are associated with worse clinical outcomes. This value may reflect the rate of physiological infarct expansion and thus serve as a tool in patient selection for IAT.

\section{INTRODUCTION/BACKGROUND}

Both time $^{1}$ and imaging ${ }^{2}$ play a significant role in the medical decision making for patients with acute ischemic strokes. Intra-arterial therapy (IAT) may be an option for patients who are either ineligible for intravenous tissue plasminogen activator (tPA), have failed tPA, or who present beyond $4.5 \mathrm{~h}$ of symptom onset. $^{3}$ Recent randomized trials, however, have failed to show the benefit of IAT versus IV $\mathrm{tPA}^{4}{ }^{5}$ thus highlighting the importance of better understanding and improving the selection processes associated with IAT. Previous studies have demonstrated the crucial importance of time with respect to endovascular efficacy, ${ }^{6}$ in which every $30 \mathrm{~min}$ delay to treatment, whether from onset to reperfusion or imaging to groin puncture, is associated with a $20 \%$ relative reduction in the probability of a good outcome. ${ }^{7}$ Although efforts to improve system processes related to IAT are already underway (ie, quality improvement registries, time metric standardization), the dynamic changes in physiology associated with treatment delays have yet to be fully understood.

The Alberta Stroke Program Early CT Score (ASPECTS) has previously been used as a tool to predict patient outcomes based on the magnitude of early ischemic changes on pretreatment CT images. Patients dichotomized to ASPECTS $\leq 7$ have been shown to possess lower rates of functional outcomes following IAT compared with those with ASPECTS $8-10 .^{10} 11$ Given the widespread availability and speed of obtaining non-contrast head CTs (NCCT), the use of the ASPECTS scoring system presents an attractive option for patient selection in IAT.

ASPECTS, however, is limited by the interpretation of a patient's pretreatment infarct at a 'static' point in time, which fails to account for the rate of infarct volume expansion that occurs during the procedure. For instance, a patient with poor collateral physiology may experience a faster deterioration of the ischemic region ${ }^{12}$ compared with other patients with similar baseline ASPECTS and treatment times, thereby resulting in a poorer outcome after endovascular reperfusion.

We hypothesized that the absolute rate of ASPECTS decay may serve as a surrogate for the rate of infarct volume expansion, thus capturing the components of time and physiology within a single parameter. In order to calculate this 'dynamic' process, we evaluated the rate of ASPECTS decline among a unique population of inter-facility transferred patients, in which initial NCCT images from the referring hospitals could be compared with repeat imaging at our facility. We further hypothesized that the rate of ASPECTS decay would not only serve as a surrogate for infarct volume expansion but also as an 
'imaging biomarker' for the prediction of patient outcomes following IAT.

\section{METHODS}

\section{Patient selection}

Institutional review board approval for the study was granted by our local institution. The study was performed as a retrospective chart review of all inter-facility transferred patients treated with IAT from six referring hospitals between December 15, 2010 and March 15, 2013. All ischemic stroke patients received IAT at a comprehensive stroke center (CSC). Patients were excluded from the analysis on the basis of: (1) posterior circulation infarctions $(\mathrm{n}=9)$; (2) absence of repeat NCCT at the treatment facility $(n=33)$; and/or (3) lost to follow-up at 90 days $(n=2)$.

\section{Data collection}

Data pertaining to baseline demographics, past medical history, admission laboratory values, and initial stroke severity (National Institutes of Health Stroke Scale (NIHSS)) were obtained through chart abstraction of the electronic medical records by research coordinators. The timing of symptom onset, initial CT imaging, repeat CT imaging, arterial access, and successful reperfusion were calculated based on the official time stamps in the patient records. Transfer time was defined as the interval between initial outside hospital $(\mathrm{OSH})$ contact with the neurointerventionist to repeat imaging at the CSC. The Totaled Health Risks in Vascular Events (THRIVE), ${ }^{13}$ Houston IAT-2 (HIAT-2), ${ }^{14}$ and Acute Physiology and Chronic Health Evaluation II (APACHE II) ${ }^{15}$ scores were calculated for all patients at both the $\mathrm{OSH}$ as well as on arrival at the treating facility. This step allowed for comparison of temporal changes in neurological impairment and medical morbidity during the inter-facility transfer process.

\section{Imaging interpretation}

All ASPECTS values were interpreted by two independent reviewers (RG and C-HJS) who were trained on ASPECTS scoring and blinded to the timing of the studies and the 90 day outcomes. A $\kappa$ score was obtained to confirm inter-rater reliability. CT images with differences in scores were reviewed by both reviewers and consensus was reached for the purposes of analysis. Information pertaining to the side of the involved hemisphere was provided for ASPECTS evaluation. Successful reperfusion was defined as a final Thrombolysis in Cerebral Infarction (TICI) score of $2 \mathrm{~b}$ or greater, as seen on carotid injection.

A third ASPECTS was obtained on the $24 \mathrm{~h}$ CT scan of all patients who achieved a reperfusion score of TICI $2 b$ or 3 . Final infarct volumes were calculated from postprocedural MRIs $(36-72 \mathrm{~h})$, as previously described. ${ }^{16}$ Symptomatic intracerebral hemorrhages were classified as any parenchymal hematoma (HI or H2) with space occupying effects. ${ }^{17}$ NIHSS deteriorations of $\geq 4$ points were not consistently captured on chart review within $24 \mathrm{~h}$ of treatment, and thus could not be included in the definition of symptomatic intracerebral hemorrhages.

\section{Rate of ASPECTS deterioration}

The absolute rate of ASPECTS decay was determined by calculating the net difference in scores between the initial image obtained at the referring facility and the repeat image performed at the CSC, divided by the change in time in hours: ((ASPECTS First $\mathrm{CT}^{-}$-ASPECTS second $\mathrm{CT}$ )/hours). The relative rate of ASPECTS decay was subsequently defined as: ((ASPECTS First $\mathrm{CT}^{-}$ASPECTS Second СT)/ASPECTS $\left.{ }_{\text {First } \mathrm{CT}}\right) /$ hours. A second rate of ASPECT deterioration was obtained considering the initial CT at the treating facility and the CT image at $24 \mathrm{~h}$ posttreatment in patients who had successful TICI $2 \mathrm{~b} / 3$ reperfusion. The time from the CT at the CSC to the end of the procedure (ie, time of successful reperfusion) was used to determine the rate of decay, as previously described. ${ }^{18}$

\section{Primary outcome measure}

'Good outcome' was defined as a 90 day modified Rankin Scale (mRS) score of $0-2$. All mRS scores were graded by a certified examiner (BAG) during follow-up examinations or telephone calls at $90 \pm 14$ days from the date of the procedure. The examiner was blinded to both the pretreatment ASPECTS scores and procedure results during the evaluation.

\section{Statistical analysis}

Each reviewer's ASPECTS value was dichotomized as either $\leq 7$ or $>8$, and cross tabulated to assess for interobserver agreement. A Cohen's $\kappa$ statistic was calculated to confirm the reliability of the independent scores. All temporal variables, including admission vital signs, APACHE II, THRIVE, HIAT-2, and ASPECTS, were collected at both the referring facility as well as the CSC. These variables were compared in a pairwise fashion using Wilcoxon signed rank tests, McNemar's tests, or paired t tests, as appropriate, in order to account for temporal changes in patient morbidity during transfer. Variables associated with good patient outcomes following IAT were identified on univariate testing $(\mathrm{p}<0.15)$, and included in a binary logistic regression model. ORs and 95\% CIs were subsequently reported. Multicollinearity testing confirmed the entry of each variable into the regression, while a Homser-Lemeshow test confirmed the 'goodness of fit' of our model.

\section{RESULTS}

We reviewed 150 consecutive stroke patients who were transferred from six referring facilities and treated with IAT at the CSC during a 27 month period. A total of 44 patients were excluded from the analysis, as described in the 'Methods'. The study cohort comprised 106 patients with a mean age of 66 \pm 14 years and an overall good outcome rate of $31 \%$. The proximal distributions of the ischemic occlusions were as follows: tandem extracranial and intracranial internal carotid artery (ICA) (14\%), isolated extracranial ICA (5\%), isolated intracranial ICA terminus (15\%), M1 middle cerebral artery (50\%), and M2 middle cerebral artery (16\%). Of the 106 patients treated with IAT, $60(57 \%)$ received IV tPA prior to inter-facility transfer.

Table 1 depicts the degree of neurological and medical morbidity for all patients at the OSHs compared with repeat assessments at the treatment facility. Median time between initial imaging at the $\mathrm{OSH}$ to repeat imaging at the CSC was $2.7 \mathrm{~h}$ (IQR 2.0-3.6), with a median last known normal to groin puncture time of $5.1 \mathrm{~h}$ (IQR 4.2-7.3) and transfer time of $1.5 \mathrm{~h}$ (IQR 1.2-1.8). In spite of these delays, there were no differences in NIHSS scores, THRIVE scores, baseline heart rate, baseline temperature, and/or APACHE II scores at the time of initial assessment compared with repeat evaluation at the CSC. ASPECTS and HIAT-2 scores, however, were significantly worse following inter-facility transfer (ASPECTS: 8 (IQR 8-9) vs 7 (IQR 7-8), p<0.001; HIAT-2: 4 (IQR 2-5) vs 5 (IQR 4-6), $\mathrm{p}<0.001)$, with a subsequent reduction in favorable ASPECTS $\geq 7(93 \%$ vs $76 \%, \mathrm{p}<0.001)$.

In the univariate analysis, good outcomes were associated with younger age, lower NIHSS scores, higher pretreatment 
Table 1 Characteristics of all intra-arterial therapy patients transferred with anterior circulation infarcts

\begin{tabular}{|c|c|c|c|}
\hline \multirow[b]{2}{*}{ Characteristic } & \multicolumn{2}{|l|}{ Transfer status } & \multirow[b]{2}{*}{$\begin{array}{l}p \\
\text { Value }\end{array}$} \\
\hline & $\begin{array}{l}\text { Assessment } \\
\text { at } 0 S H \\
(n=106)\end{array}$ & $\begin{array}{l}\text { Assessment at } \\
\text { CSC following } \\
\text { transfer } \\
(n=106)\end{array}$ & \\
\hline \multicolumn{4}{|l|}{ Neurological assessment } \\
\hline NIHSS (median (IQR)) & $19(15-23)$ & $19(15-24)$ & 0.943 \\
\hline THRIVE score (median (IQR)) & $5(4-6)$ & $5(4-6)$ & 0.513 \\
\hline HIAT-2 score (median (IQR)) & $4(2-5)$ & $5(4-6)$ & $<0.001$ \\
\hline \multicolumn{4}{|l|}{ Medical assessment } \\
\hline Admission MAP (mean (SD)) & $102.3(17.3)$ & $92.8(18.0)$ & $<0.001$ \\
\hline Admission HR (mean (SD)) & $83.8(21.5)$ & $80.9(18.0)$ & 0.202 \\
\hline $\begin{array}{l}\text { Admission temperature (mean } \\
\text { (SD)) }\end{array}$ & $36.5(0.53)$ & $36.4(0.84)$ & 0.343 \\
\hline APACHE II score (median (IQR)) & $12(10-14)$ & $12(10-14)$ & 0.403 \\
\hline \multicolumn{4}{|l|}{ Imaging studies } \\
\hline $\begin{array}{l}\text { ASPECTS on initial imaging } \\
\text { (median (IQR)) }\end{array}$ & $8(8-9)$ & $7(7-8)$ & $<0.001$ \\
\hline $\begin{array}{l}\text { ASPECTS } \geq 7 \text { on initial imaging } \\
(\mathrm{n}(\%))\end{array}$ & $98(93)$ & $80(76)$ & $<0.001$ \\
\hline \multicolumn{4}{|c|}{$\begin{array}{l}\text { APACHE II, Acute Physiology and Chronic Health Evaluation II; ASPECTS, Alberta } \\
\text { Stroke Program Early CT Score; CSC, comprehensive stroke center; HIAT-2, Houston } \\
\text { Intra-Arterial Therapy 2; HR, heart rate; MAP, mean arterial pressure; NIHSS, National } \\
\text { Institutes of Health Stroke Scale; OSH, outside hospital; THRIVE, Totaled Health Risks } \\
\text { in Vascular Events. }\end{array}$} \\
\hline
\end{tabular}

ASPECTS, successful reperfusion, smaller postprocedure infarct volumes, and absence of symptomatic hemorrhages (table 2). Patients with favorable outcomes also had significantly lower rates of both absolute ASPECTS decay $(0.14 \pm 0.23$ score/h vs $0.49 \pm 0.39$ score $/ \mathrm{h} ; \mathrm{p}<0.001)$ and relative ASPECTS decay $(0.018 \pm 0.03 \%$ score $/ \mathrm{h}$ vs $0.079 \pm 0.075 \%$ score $/ \mathrm{h} ; \mathrm{p}<0.001)$ compared with those with poorer outcomes, as measured from the initial OSH CT to the pretreatment CSC CT.

In a binary logistic regression model adjusting for age, NIHSS, reperfusion success, and symptomatic hemorrhage, the absolute rate of ASPECTS decay (OR 0.043 ; 95\% CI 0.004 to $0.471 ; \mathrm{p}=0.01)$ was a stronger predictor of patient outcome than static pretreatment ASPECTS (OR 0.64; 95\% CI 0.38 to $1.04 ; \mathrm{p}=0.075$ ) (table 3 ). As shown in figure 1 , the OR for a good outcome declined in a stepwise fashion as the rate of ASPECTS deterioration increased from $<0.2$ points $/$ h to $>0.6$ points/h (figure 1). In particular, among the 44 patients with favorable ASPECTS (8-10) observed on CSC imaging immediately prior to IAT, patients with poor outcomes had significantly faster rates of absolute ASPECTS decay compared with those with favorable outcomes (poor: $0.31 \pm 0.32$ score/h vs good: $0.12 \pm 0.22$ score $/ \mathrm{h}, \mathrm{p}=0.02$ ).

In a secondary analysis of all patients with successful reperfusion $(n=83)$, the rate of ASPECTS decay between immediate pretreatment CT and post-treatment CT obtained within $24 \mathrm{~h}$ of reperfusion was similarly associated with 90 day outcomes. Patients with favorable outcomes had lower rates of absolute ASPECTS deterioration compared with those with unfavorable outcomes $(0.27 \pm 0.46 \mathrm{score} / \mathrm{h}$ vs $0.90 \pm 0.99 \mathrm{score} / \mathrm{h} ; \mathrm{p}<0.001)$. Twenty-three patients did not have successful reperfusion, and thus could not be included in the secondary analysis.

The $\kappa$ score for interobserver agreement in ASPECTS was 0.80 . All interpretations were measured with prior knowledge of the involved hemisphere.
Table 2 Characteristics of all transferred patients with anterior circulation infarcts treated with intra-arterial therapy

\begin{tabular}{|c|c|c|c|}
\hline \multirow[b]{2}{*}{ Characteristic } & \multicolumn{2}{|l|}{90 day outcome } & \multirow[b]{2}{*}{ p Value } \\
\hline & $\begin{array}{l}\text { Good (mRS 0-2) } \\
(n=33)\end{array}$ & $\begin{array}{l}\text { Poor (mRS 3-6) } \\
(n=73)\end{array}$ & \\
\hline \multicolumn{4}{|l|}{ Demographics } \\
\hline Age (mean (SD)) & $59.0(12.4)$ & $69.4(13.0)$ & $<0.001$ \\
\hline Male sex (n (\%)) & $21(64)$ & $39(53)$ & 0.326 \\
\hline Hypertension (n (\%)) & $25(76)$ & $47(64)$ & 0.245 \\
\hline Atrial fibrillation (n (\%)) & $11(33)$ & $26(36)$ & 0.819 \\
\hline \multicolumn{4}{|l|}{ Laboratory values } \\
\hline HgbA1c (mean (SD)) & $5.9(1.2)$ & $6.3(1.3)$ & 0.150 \\
\hline LDL (mean (SD)) & $87.6(31.7)$ & $91.2(39.2)$ & 0.648 \\
\hline \multicolumn{4}{|l|}{ Neurological assessment } \\
\hline $\begin{array}{l}\text { Pretreatment NIHSS (median } \\
(\text { IQR)) }\end{array}$ & $17(12-20)$ & $20(17-24)$ & $<0.001$ \\
\hline \multicolumn{4}{|l|}{ Imaging parameters } \\
\hline $\begin{array}{l}\text { Pretreatment ASPECTS (median } \\
(\mathrm{IQR}) \text { ) }\end{array}$ & $8(7-9)$ & $7(6-8)$ & $<0.001$ \\
\hline $\begin{array}{l}\text { Successful reperfusion, } \mathrm{TICl} \\
\geq 2 \mathrm{~b}(\mathrm{n}(\%))\end{array}$ & $32(97)$ & $51(70)$ & 0.002 \\
\hline $\begin{array}{l}\text { Postprocedure infarct volume } \\
\left(\mathrm{cm}^{3}\right) \text { (median (IQR)) }\end{array}$ & $21(10-38)$ & $91(31-135)$ & $<0.001$ \\
\hline \multicolumn{4}{|l|}{ OSH CT to pretreatment CSC CT } \\
\hline $\begin{array}{l}\text { Rate of ASPECTS decline } \\
\text { ( } \Delta \text { score/h) (mean (SD)) }\end{array}$ & $0.14(0.23)$ & $0.49(0.39)$ & $<0.001$ \\
\hline $\begin{array}{l}\text { Relative rate of ASPECTS } \\
\text { decline }(\triangle \mathrm{ASPECTS/ASPECTS}) / \mathrm{h} \\
\text { (mean }(\mathrm{SD}) \text { ) }\end{array}$ & $0.018(0.03)$ & $0.079(0.075)$ & $<0.001$ \\
\hline \multicolumn{4}{|l|}{ Treatment times (min) } \\
\hline $\begin{array}{l}\text { LKN to groin puncture (median } \\
\text { (IQR)) }\end{array}$ & $305(255-421)$ & $308(255-418)$ & 0.926 \\
\hline Procedure time (median (IQR)) & $67(44-100)$ & $77(51-109)$ & 0.260 \\
\hline \multicolumn{4}{|l|}{ Complications } \\
\hline $\begin{array}{l}\text { Symptomatic hemorrhage } \\
\text { PH1/2 (n (\%)) }\end{array}$ & $1(3)$ & $12(16)$ & 0.051 \\
\hline
\end{tabular}

ASPECTS, Alberta Stroke Program Early CT Score; CSC, comprehensive stroke center LDL, low density lipoprotein; LKN, last known normal; mRS, modified Rankin Scale; NIHSS, National Institutes of Health Stroke Scale; OSH, outside hospital; PH, parenchymal hematoma; $\mathrm{TICl}$, Thrombolysis in Cerebral Infarction.

\section{DISCUSSION}

Elapsed time to reperfusion is an important determinant of clinical outcomes after IAT. $^{8}$ Additionally, the final infarct volume after IAT appears to be a potent surrogate for estimating clinical outcomes at an earlier time point. ${ }^{19}$ Patients who are transferred from outside facilities reflect a unique cohort in whom two CT

Table 3 Binary logistic regression model identifying predictors associated with good patient outcomes following endovascular therapy for ischemic stroke

\begin{tabular}{lll}
\hline Variable & OR $(95 \% \mathrm{CI})$ & p Value \\
\hline Age & $0.92(0.88-0.98)$ & 0.004 \\
Successful reperfusion & $0.67(0.006-0.712)$ & 0.025 \\
Rate of ASPECTS decay (Dunit score/h) & $0.043(0.004-0.471)$ & 0.010 \\
NIHSS score & $0.91(0.82-1.00)$ & 0.061 \\
Pretreatment ASPECTS (per unit decline) & $0.64(0.38-1.04)$ & 0.075 \\
Symptomatic hemorrhage & $3.15(0.30-33.4)$ & 0.340 \\
\hline
\end{tabular}

Homser-Lemeshow test depicts goodness of fit to the model $\left(\chi^{2}=9.6 ; p=0.29\right)$.

ASPECTS, Alberta Stroke Program Early CT Score; NIHSS, National Institutes of Health Stroke Scale. 
Association Between Rate of ASPECTS Decay and Good Outcomes

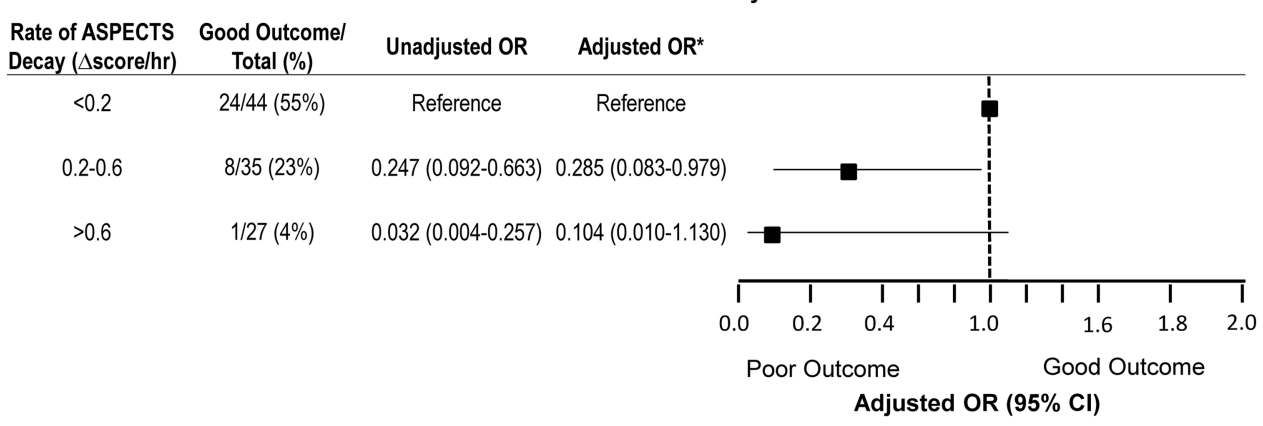

Figure 1 Association between rate of Alberta Stroke Program Early CT Score (ASPECTS) decay and good outcomes. The model has been adjusted for age, National Institutes of Health Stroke Scale, reperfusion success, ASPECTS, and symptomatic hemorrhage.

images are typically obtained prior to proceeding to IAT. The association between pretreatment core infarct volume on MRI or ASPECTS on CT to good clinical outcomes has been well established. ${ }^{20}$ Unfortunately, these images are static and do not incorporate the subsequent time delays that occur to achieve reperfusion during which further infarct expansion occurs. The current analysis shows that the change in ASPECTS may be more important than the static pretreatment ASPECTS in patients who are transferred. Moreover, this reiterates the impact of time on infarct expansion and the importance of rapid reperfusion to overcome this process.

With the recent series of negative clinical trials surrounding $\mathrm{IAT}^{4}{ }^{5}$ there has been more attention focused on patient selection and process improvement to more rapid reperfusion. The pretreatment core infarct appears to correlate with the patient's ultimate clinical outcome. Advocates of multimodal imaging through $\mathrm{MRI}^{21}$ or CT perfusion ${ }^{22}$ have defined thresholds that may identify ideal patients. Unfortunately, even with such tools, more than $50 \%$ of patients do not achieve a good outcome. ${ }^{2}$ Moreover, recent data have shown that time delays are directly proportional to the probability of a poor outcome. ${ }^{6-8}$ The time from when the pretreatment image has been obtained to reperfusion can represent a substantial amount of time during which further infarct burden has been accrued. The dynamic process of time and infarct expansion is thus not captured during current imaging paradigms, except for patients who are transferred.

Inter-facility transfer patients appear to have worse clinical outcomes compared with patients presenting in the emergency room of the treating facility. ${ }^{7}$ Our current analysis points to the rate of infarct expansion, as quantified by the absolute rate of ASPECTS decline, as being a potent biomarker for assessing such patients. In particular, the OR for a good outcome was 0.043 for every unit increase in absolute ASPECTS decay per hour. In practical terms, a patient whose ASPECTS score decreases by 2 points per hour (ie, 10 to a 6 in $120 \mathrm{~min}$ ) will have a 23 -fold lower probability for a good outcome than an individual whose ASPECTS declines at a rate of 1 point per hour (ie, 10 to an 8 in $120 \mathrm{~min}$ ). As such, the utilization of absolute ASPECTS decay in patient selection may hold clinical relevance in determining patients who may maximally benefit from intervention, regardless of pretreatment ASPECTS or initial stroke severity.

This current model helps us to understand the complex interplay between time and ischemic burden. For example, patients who present with an ASPECTS decay of $<0.2$ points per hour not only have the highest probability of a good outcome $(55 \%)$ but may represent a subgroup of individuals whose superior physiology protects the brain from infarct volume expansion and, thus, reduces their vulnerability to treatment delays.

To our knowledge, the integration of a 'dynamic' variable in the patient selection process for IAT in inter-facility transfers has yet to be included in any scoring system to date (ie, THRIVE, HIAT-2). According to our analysis, the current metrics of NIHSS, THRIVE, and APACHE II did not differ over time and thus fail to capture the changes in neurological morbidity that occur over time. In contrast, our ASPECTS model successfully captures the change in infarct volume expansion over time, thereby providing a measure for the decline in neurological morbidity that correlates to patient outcomes.

Our present study has several limitations. First, given the retrospective nature of the analysis, OSH imaging data could only be obtained from the six contributing hospitals. As a result, consecutive patients treated at our facility who were transferred from other centers were not included in the analysis, creating a potential selection bias. Second, as a single center study, the results and conclusions may not be applicable to other centers, in which differences in treatment times and transfer protocols vary. Third, given that ASPECTS values are weighted heavily towards the location of the infarct, rather than the total volume, we recognize that such scores can only serve as a 'surrogate' for infarct volume expansion and do not reflect the true volume of the core infarct. Fourth, there may be technique and quality differences in the CT scanners at outside facilities compared with the images obtained at our institution. This may lead to potential differences in ASPECT scoring. Lastly, despite the absolute rate of decay being small, it highlights that a static ASPECT score may not reflect the rate of tissue infarct expansion as may be estimated in inter-facility transfer patients.

In conclusion, our study demonstrates that the rate of ASPECTS decay during inter-facility transfers for ischemic strokes is not only associated with patient outcomes following IAT, but is also a stronger predictor of functional independence than the static pretreatment ASPECTS. In the absence of obtaining sequential multimodal images (ie, two or more MRIs), the rate of ASPECTS decay may serve as a functional surrogate for the rate of infarct volume expansion. In the setting of real time delays in system processes associated with inter-facility transfers, this metric may be of particular value in identifying patients who may or may not derive benefit from intervention based on their underlying physiology and susceptibility to ischemic burden.

\section{Author affiliations}

'Department of Neurology, Emory University School of Medicine, Atlanta, Georgia, USA 
${ }^{2}$ Marcus Stroke and Neuroscience Center, Grady Memorial Hospital, Atlanta, Georgia, USA

${ }^{3}$ Wellstar Health System, Marietta, Georgia, USA

$v^{4}$ Southern Regional Medical Center, Riverdale, Georgia, USA

${ }^{5}$ Atlanta Medical Center, Atlanta, Georgia, USA

${ }^{6}$ Gwinnett Medical Center, Gwinnett, Georgia, USA

${ }^{7}$ Northside Hospital, Atlanta, Georgia, USA

Correction notice This article has been corrected since it was published Online First. Table 3 has been amended.

Contributors Writing of manuscript: C-HJS and RG. Conception of research: C-HJS, $R G$, and RGN. Revision of manuscript: MRF, SRB, AMA, FN, and MY. Data collection: KC, BAG, SZ, KA, DC, SG, HP, and ME. Data analysis and figures: C-HJS and RG.

Competing interests RGN serves on the scientific advisory board for Stryker Neurovascular, Covidien, and CoAxia. He also serves on the Data Safety Monitoring Board for Rapid Medical and Imaging Core Lab for Covidien. He is the editor of Interventional Neurology. RG serves on the scientific advisory board for Stryker Neurovascular, Covidien, and CoAxia. He is also a member of the Data Safety Monitoring Board for Reverse Medical and Rapid Medical. He is an associate editor for the Journal of Neuroimaging and associate editor for Interventional Neurology. SRB serves as a consultant for Neural Stem.

Ethics approval The study was approved by the local institutional review board.

Provenance and peer review Not commissioned; externally peer reviewed.

Data sharing statement The unpublished data from this dataset is held by Grady Memorial Hospital and RGN. Any requests for data sharing would be required to be discussed with him directly.

\section{REFERENCES}

1 Adams H Jr, del Zoppo G, Alberts MJ, et al. Guidelines for the early management of adults with ischemic stroke. Stroke 2007;38:1655-711.

2 Lansberg MG, Straka M, Kemp S, et al. DEFUSE 2 study investigators. MRI profile and response to endovascular reperfusion after stroke (DEFUSE 2): a prospective cohort study. Lancet Neurol 2012;11:860-7.

3 Hacke W, Kaste M, Bluhmki E, et al. Thrombolysis with alteplase 3 to 4.5 hours after acute ischemic stroke. N Engl I Med 2008:359:1317-29.

4 Ciccone A, Valvassori L, Nichelatti M, et al. SYNTHESIS Expansion Investigators. Endovascular treatment for acute ischemic stroke. $N$ Engl I Med 2013;368:904-13.

5 Broderick JP, Palesch YY, Demchuk AM, et al. Endovascular therapy after intravenous t-PA versus t-PA alone for stroke. N Engl J Med 2013;368:893-903.

6 Khatri P, Abruzzo T, Yeatts SD, et al.; IMS I and II Investigators. Good clinical outcome after ischemic stroke with successful revascularization is time-dependent. Neurology 2009;73:1066-72.
7 Sun $\mathrm{CH}$, Nogueira RG, Glenn BA, et al. "Picture to puncture": a novel time metric to enhance outcomes in patients transferred for endovascular reperfusion in acute ischemic stroke. Circulation 2013;127:1139-48.

8 Mazighi M, Chaudhry SA, Ribo M, et al. Impact of onset-to-reperfusion time on stroke mortality: a collaborative pooled analysis. Circulation 2013;127:1980-5.

9 Barber PA, Demchuk A, Zhang J, et al. Validity and reliability of a quantitative computed tomography score in predicting outcome of hyperacute stroke before thrombolytic therapy. ASPECTS Study Group. Alberta Stroke Programme Early CT Score. Lancet 2000;355:1670-4.

10 Hill MD, Rowley HA, Adler F, et al. Selection of acute ischemic stroke patients for intra-arterial thrombolysis with pro-urokinase by using ASPECTS. Stroke 2003;34:1925-31.

11 Hill MD, Demchuk AM, Tomsick TA, et al. Using the baseline CT scan to select acute stroke patients for IV-IA therapy. AJNR 2006;27:1612-16.

12 Miteff $F$, Levi CR, Bateman GA, et al. The independent predictive utility of computed tomography angiographic collateral status in acute ischaemic stroke. Brain 2009;132:2231-8.

13 Flint AC, Cullen SP, Faigeles BS, et al. Predicting long-term outcome after endovascular stroke treatment: the Totaled Health Risks in Vascular Events score. Am J Neuroradiol 2010;31:1192-6.

14 Hen $\mathrm{H}$, Barreto $A D$, Liebeskind DS, et al. Identifying patients at high risk for poor outcome after intra-arterial therapy for acute ischemic stroke. Stroke 2009;40:1780-5.

15 Knaus WA, Draper EA, Wagner DP, et al. APACHE II: a severity of disease classification system. Crit Care Med 1985;13:818-29.

16 Rangaraju S, Owada K, Noorian AR, et al. Comparison of final infarct volumes in patients who received endovascular therapy or intravenous thrombolysis for acute intracranial large-vessel occlusions. JAMA Neuro 2013;70:831-6.

17 Berger C, Fiorelli M, Steiner T, et al. Hemorrhagic transformation of ischemic brain tissue: asymptomatic or symptomatic? Stroke 2001:32:1330-5.

18 Almekhlafi MA, Eesa M, Menon BK, et al. Ultrashort imaging to reperfusion time interval arrests core expansion in endovascular therapy for acute ischemic stroke. J Neurointerv Surg 2013;5(Suppl 1):i58-61.

19 Zaidi SF, Aghaebrahim A, Urra X, et al. Final infarct volume is a stronger predictor of outcome than recanalization in patients with proximal middle cerebral artery occlusion treated with endovascular therapy. Stroke 2012;43:3238-44.

20 Goyal M, Menon BK, Coutts SB, et al. Penumbra Pivotal Stroke Trial Investigators, Calgary Stroke Program, and the Seaman MR Research Center. Effect of baseline CT scan appearance and time to recanalization on clinical outcomes in endovascular thrombectomy of acute ischemic strokes. Stroke 2011;42:93-7.

21 Yoo AJ, Verduzco LA, Schaefer PW, et al. MRI-based selection for intra-arterial stroke therapy: value of pretreatment diffusion-weighted imaging lesion volume in selecting patients with acute stroke who will benefit from early recanalization. Stroke 2009;40:2046-54.

22 Turk AS, Magarick JA, Frei D, et al. CT perfusion-guided patient selection for endovascular recanalization in acute ischemic stroke: a multicenter study. J Neurointerv Surg 2013;5:523-7. 